\title{
The Accutension Stetho, an automated auscultatory device to validate automated sphygmomanometer readings in individual patients
}

\author{
Bruce S. Alpert ${ }^{1}$
}

Received: 12 January 2018 / Revised: 20 February 2018 / Accepted: 26 February 2018 / Published online: 6 April 2018

(c) The Author(s) 2018. This article is published with open access

\begin{abstract}
The aim of this report is to describe a new device that can validate, by automated auscultation, individual blood pressure (BP) readings taken by automated sphygmomanometers. The Accutension Stetho utilizes a smartphone application in conjunction with a specially designed stethoscope that interfaces directly into the smartphone via the earphone jack. The Korotkoff sounds are recorded by the application and are analyzed by the operator on the screen of the smartphone simultaneously with the images from the sphygmomanometer screen during BP estimation. Current auscultatory validation standards require at least 85 subjects and strict statistical criteria for passage. A device that passes can make no guarantee of accuracy on individual patients. The Accutension Stetho is an inexpensive smartphone/stethoscope kit combination that estimates precise BP values by auscultation to confirm the accuracy of an automated sphygmomanometer's readings on individual patients. This should be of great value for both professional and, in certain circumstances, self-measurement BP. Patients will avoid both unnecessary treatment and errors of underestimation of BP, in which the patient requires therapy. The Stetho's software has been validated in an independent ANSI/AAMI/ISO standard study. The Stetho has been shown to perform without difficulty in multiple deflation-based devices by many manufacturers.
\end{abstract}

Data from the Centers for Disease Control demonstrate that at least 67 million American adults have high blood pressure (BP) [1]. The diagnosis and treatment of hypertension and many other clinical conditions in which BP can be altered is dependent upon the accurate estimation of BP by indirect techniques such as oscillometry and auscultation. There are many automated sphygmomanometers available on the market, but even if the device has passed the United States National Standard protocol [2] there is no assurance that a particular device will give accurate BP readings in individual patients. To pass the auscultatory validation

Bruce S. Alpert: Retired from University of Tennessee Health Science Center.

Electronic supplementary material The online version of this article (https://doi.org/10.1038/s41371-018-0053-2) contains supplementary material, which is available to authorized users.

Bruce S. Alpert

bsa2347@gmail.com

1 Department of Pediatrics, University of Tennessee Health Science Center (retired), Memphis, TN, USA requirements the manufacturer must test at least 85 subjects, with a wide range of $\mathrm{BP}$ values needed. There are two strict statistical analyses that ensure that few readings deviate from the "gold standard" manual auscultation readings. The first criterion states that the mean \pm standard deviation (SD) of at least 255 paired manual/device comparisons be less than or equal to $5 \pm 8 \mathrm{mmHg}$. If one considers readings within only $2 \mathrm{SD}$ values, then acceptable device values may be $16 \mathrm{mmHg}$ higher, or $16 \mathrm{mmHg}$ lower than the manual value. Clinicians require much more precision. It would be of great value if there is a device that could assess accuracy of automated sphygmomanometer readings on individual patients. This manuscript reports such a device. Data on validation of the app software and the generalizability of the device are also included.

The Accutension Stetho is a novel device that operates by use of a smartphone application and an inexpensive ( $<\$ 30$ U.S.) kit containing a specially designed stethoscope and required connectors. This report will describe the design and operation of this device.

The Accutension Stetho comes as a kit for the automated auscultatory calibration of automated sphygmomanometer (BPM) readings. The initial step is to download the 
"Accutension Stetho" app to a smartphone. The app contains detailed instructions/diagrams for ease of use.

Figure 1 shows the set-up for an individual patient performing the calibration of BP values from his/her selfmeasurement sphygmomanometer. When this procedure takes place in a medical environment the trained aide/nurse/ physician would hold the smartphone. Note that the arm on which the BP is being measured is supported at heart level and the patient has back support. The patient could easily be taught to do the recording process with the trained medical personnel doing the analysis.

Figure 2 shows the structure of the device and demonstrates a frame of the video image on the smartphone screen

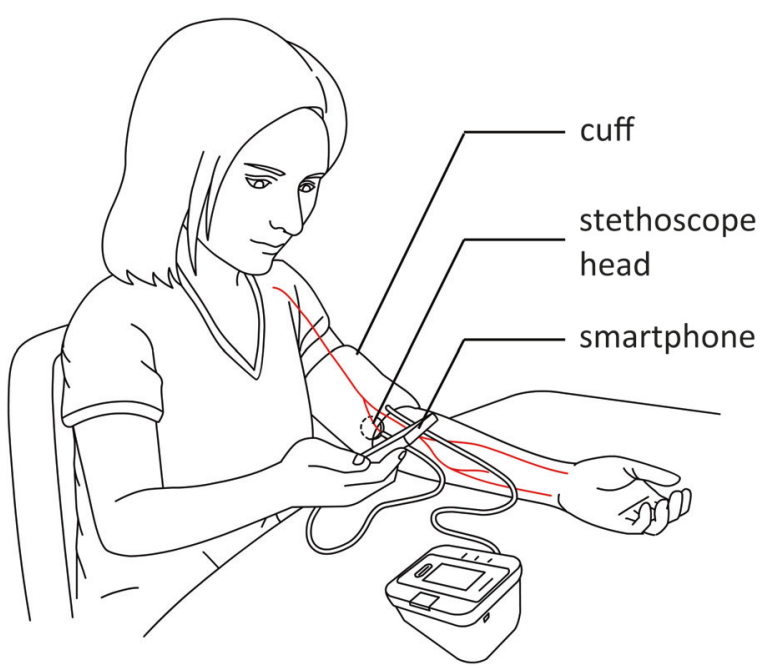

Fig. 1 Setup and use of Accutension Stetho in a BPM self-validation during operation. The simple device hardware is made of a traditional acoustic stethoscope head (chest piece) that records Korotkoff sounds, an air tube that conducts the sounds, a microphone that converts the sounds to electrical signal, and a $3.5 \mathrm{~mm}$ earphone plug that transmits the electrical signal into the smartphone. During operation, the BPM is cycled, and at the end of inflation the video is begun. The patient or medical person (operator) records the BPM screen during the deflation phase. Once the deflation is complete the operator presses the stop button on the app. Note that during the BP estimation the app displays the Korotkoff sound signals and pattern. In addition, the operator may choose to listen to the Korotkoff sounds to confirm that the vertical spikes are the true Korotkoff sounds.

Figure 3 displays a typical set of Korotkoff sounds [3], oscillometric pulses, and cuff pressure during a single deflation. The smartphone screen displays the Korotkoff sound profile with one vertical line that is manually movable by touch of the screen. The operator slides the vertical line to coincide with the first sharp upward spike (K1); this is the systolic BP value. Then the line is moved to coincide with the last significant sound peak (K5); this is the diastolic BP value. The app then selects the precise BP value that was displayed on the BPM screen at the time of these two selected points. If the BPM uses a continuous deflation rate the BP value on the screen will be the cuff pressure value precisely at the oscillometric spike (at the time of each Korotkoff sound deflection). If the device uses step-deflations, the value on the BPM screen will be within a few $\mathrm{mmHg}$ of the pressure within the cuff.
Fig. 2 Structure of Accutension Stetho: a stethoscope consisting of a stethoscope head, a tube and a microphone with an earphone plug and an app running on the smartphone

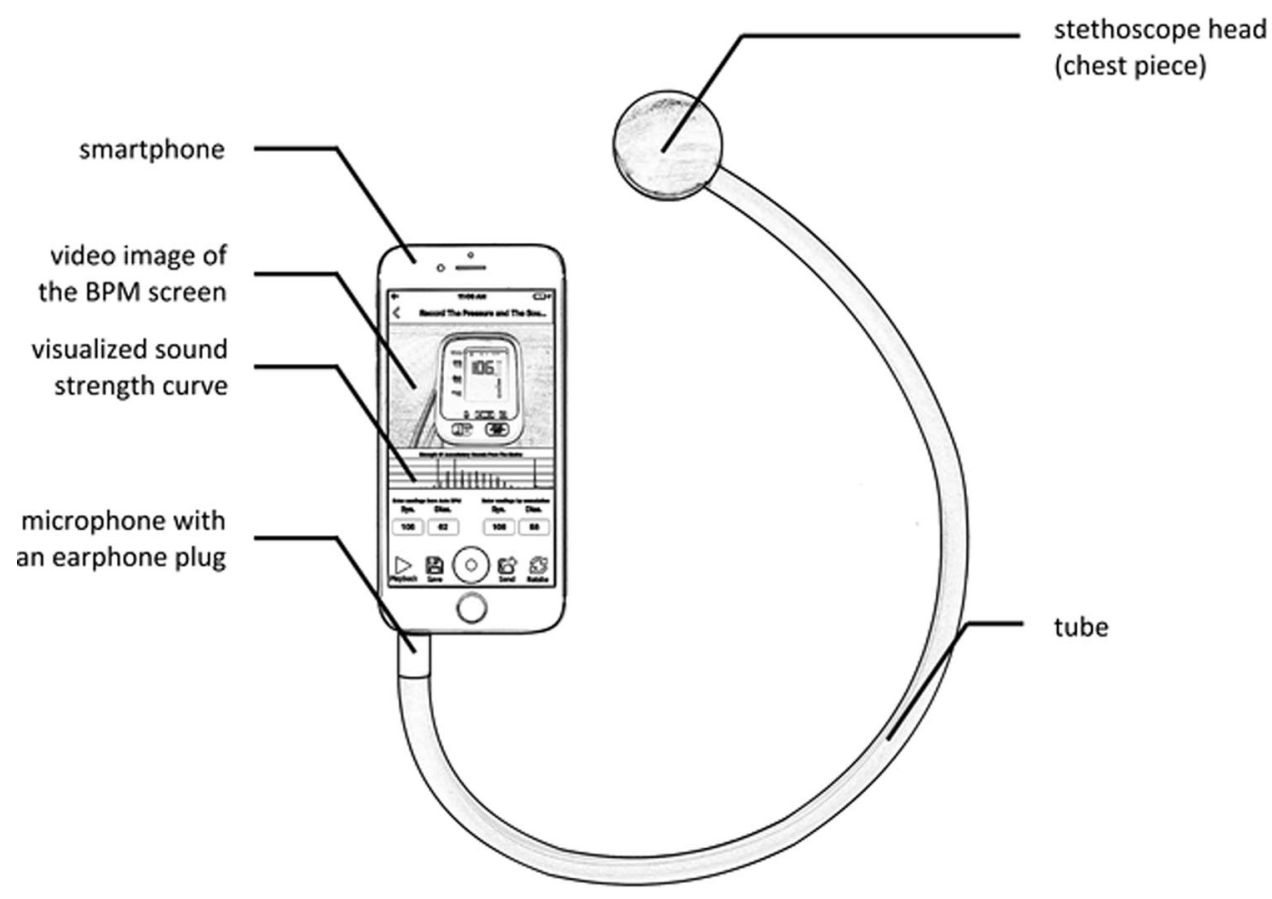


Fig. 3 Korotkoff sounds strength, cuff pressure oscillation and cuff pressure vs. time during cuff deflation from a BPM. The dots mark the peak of Korotkoff sounds and the peak of cuff pressure oscillation, respectively, and they coincide with each other. An automated BPM refreshes the cuff pressure on its screen when it detects the cuff pressure oscillation peak
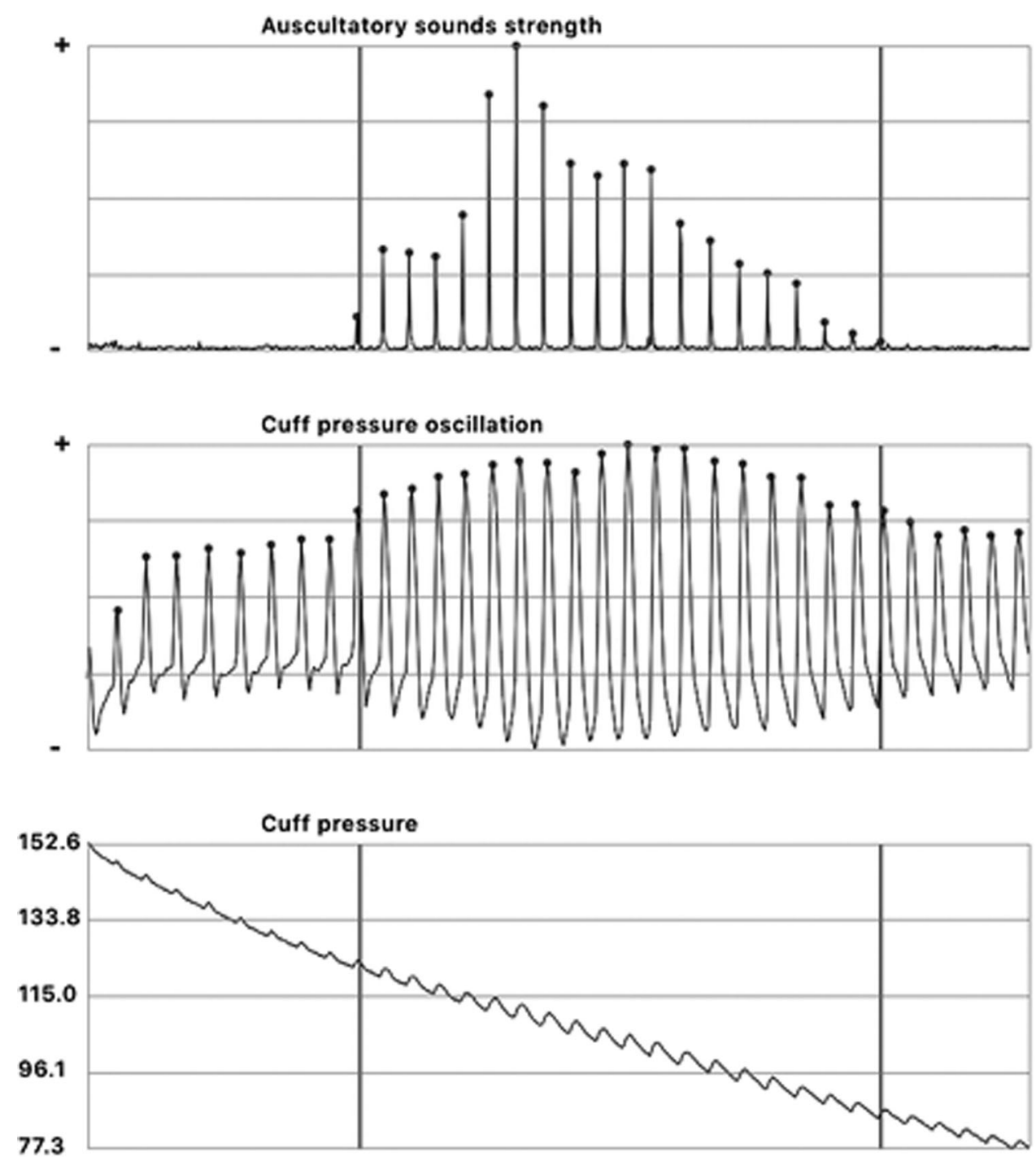

The BPM reading for an individual cycle is then compared to the reading estimated by the Accutension Stetho software. Because the Accutension detection system is by auscultation, the value will be virtually identical to the value that would have been recorded by a trained observer performing the ANSI/AAMI/ISO validation procedure [2]. A short video is attached to aid in the reader's understanding of the operation of the process.

A recent publication by Chu et al. [4] reported the validation to the ANSI/AAMI/ISO Standard [2] requirements of an Accutension auscultatory sphygmomanometer, which is not the same device as the one described here, that uses the same software algorithm as the Accutension Stetho. The ANSI/AAMI/ISO Standard uses two methods for statistical analysis of the validation data for an automated sphygmomanometer. The mean \pm SD for Method 1 (255 device minus average of two observers' estimates) were $2.45 \pm$ $2.24 \mathrm{mmHg}$ for systolic BP and $0.69 \pm 2.09 \mathrm{mmHg}$ for diastolic BP. The Method 2 data $(85$ pooled individual patient data) showed $2.45 \pm 1.47 \mathrm{mmHg}$ for systolic BP and $0.69 \pm 1.36 \mathrm{mmHg}$ for diastolic BP. All of these values were well within the passing requirements of the Standard. The excellent correlation was in part due to the comparison of $\mathrm{BP}$ values recorded from manual auscultation to "simultaneous" values recorded by an automated cell phone app that also uses auscultation.

A possible limitation of the Accutension Stetho is whether it will function accurately with automated BPMs currently marketed. Table 1 displays the manufacturers and model numbers of the devices that have been tested and found to function without difficulty. The performance on deflation-based technology was consistently excellent because, even though the BPM screen displays only intermittent readings suggesting step-deflation, the software performs, in fact, linear deflation. The BP values appearing on the BPM screen are the displays of the cuff pressures at the time of an oscillometric spike, which coincides with the Korotkoff sound. The device has not been able to function 
Table 1 Devices with successful testing with the Accutension Stetho

\begin{tabular}{llll}
\hline Brand & Model & $\begin{array}{l}\text { Measure during } \\
\text { deflation }\end{array}$ & $\begin{array}{l}\text { Linear } \\
\text { deflation }\end{array}$ \\
\hline Omron & BP786 & Yes & Yes \\
& BP760N & Yes & Yes \\
& HEM-7051 & Yes & Yes \\
& HEM-7111 & Yes & Yes \\
& HEM-J12 & Yes & Yes \\
MABIS & M3 & Yes & Yes \\
HEALTHCARE & 04-596-008 & Yes & Yes \\
HOMEDICS & BPA-060 & Yes & Yes \\
Beurer & BM26 & Yes & Yes \\
LifeSource & UA-767 & Yes & Yes \\
ReliOn & BP200 & Yes & Yes \\
Microlife & 3MC1-PC & Yes & Yes \\
AND & UA705 & Yes & Yes \\
Panasonic & EW3108WQ & No & N/A
\end{tabular}

${ }^{\mathrm{a}}$ The Panasonic device estimates BP using inflation-based technology

when using an inflation-based technology. This issue is caused by the noise of the pump interfering with the Korotkoff sound recordings.

Automated sphygmomanometers most commonly utilize oscillometric technology. By passing the ANSI/AAMI/ISO Standard testing [2] the device can deliver accurate BP estimates to large populations. There are many variables that affect the validity of individual BP estimation, including arterial compliance, BP level, arm composition, and arm shape [3]. No manufacturer can factor into his/her algorithm corrections that would ensure BP accuracy for all patients. Thus, a device such as the Accutension Stetho will be a critical addition to the initial use of a specific BPM in a specific patient. Once a BPM has been proven to give accurate $\mathrm{BP}$ values the medical team can have confidence that the readings can be used for both diagnosis and treatment, if needed, for a large variety of medical conditions. Periodic revalidation should be performed to ensure that any changes in the patient's arterial properties do not lead to BP inaccuracy. The Accutension Stetho is very inexpensive, requires minimal training, and allows medical personnel or, in some instances, the trained patient, to select a BPM for his/her care. The software algorithm utilized within the Stetho device has been validated in an independent study to meet the ANSI/AAMI/ISO Standard requirements [4]. Testing on multiple devices from multiple manufacturers has shown generalizability to deflation-based BPMs (Table 1). To date, the Stetho device has not been shown to be useful with inflation-based technology because the noise of the pump obliterates the $\mathrm{K}$ sounds.
The Accutension Stetho cannot, of course, correct for errors made by the medical personnel or the patient with respect to factors such as cuff size, failure to rest prior to initiating a reading, lack of arm and/or back support, etc.

The routine use of the Stetho device to ensure the accuracy of automated sphygmomanometers will improve clinical care in two ways: (1) avoiding treatment, and its possible side effects, in patients whose BP is incorrectly estimated by an automated device to be elevated, when, in fact, it is normal; (2) giving needed treatment to patients in whom the BP is incorrectly estimated by an automated device to be normal, when, in fact, it is elevated.

\section{Summary}

\section{What is known about topic?}

- Automated blood pressure estimation now the most commonly used methodology

- Individual blood pressure devices validated to be accurate on large populations

- Proprietary algorithms in use do not ensure accuracy for individual patients

\section{What this study adds?}

- New smartphone app with digital stethoscope available to do automated auscultation to validate blood pressure monitor readings on individual patients

- New device critical in both clinical care for diagnosis and therapy and in research populations

- Device inexpensive and can be used by patients themselves

Acknowledgements The Accutension Stetho device was provided by Dr. Junfeng Zhao. Dr. Alpert is greatly indebted to Andrea Patters for the review and submission of the manuscript.

\section{Compliance with ethical standards}

Conflict of interest The authors declare that they have no conflict of interest.

Open Access This article is licensed under a Creative Commons Attribution 4.0 International License, which permits use, sharing, adaptation, distribution and reproduction in any medium or format, as long as you give appropriate credit to the original author(s) and the source, provide a link to the Creative Commons license, and indicate if changes were made. The images or other third party material in this article are included in the article's Creative Commons license, unless indicated otherwise in a credit line to the material. If material is not included in the article's Creative Commons license and your intended use is not permitted by statutory regulation or exceeds the permitted use, you will need to obtain permission directly from the copyright 
holder. To view a copy of this license, visit http://creativecommons. org/licenses/by/4.0/.

\section{References}

1. Centers for Disease and Prevention. U.S. Department of Health \& Human Services (2104). https://www.cdc.gov/bloodpressure/ infographic.htm. Accessed September 2017.

2. Association for the Advancement of Medical Instrumentation. Noninvasive Sphygmomanometers - Part 2: clinical investigation of automated measurement type ANSI/AAMI/ISO 81060-2/ANSIAAMI, 2nd edn. Arlington, VA: AAMI 2013.
3. Pickering TG, Hall JE, Appel LJ, Falkner BE, Graves J, Hill MN, et al. Recommendations for blood pressure measurement in humans and experimental animals: Part 1: Blood pressure measurement in humans: a statement for professionals from the Subcommittee of Professional and Public Education of the American Heart Association Council on High Blood Pressure Research. Circulation. 2005; 111:697-716.

4. Chu G, Zhang Z, Xu M, Huang D, Dai Q. Validation of a smartphone auscultatory blood pressure kit Accutension XYZ-110 in adults according to the ANSI/AAMI/ISO 81060-2: 2013 standard. Blood Press Monit. 2017;22:290-4. 